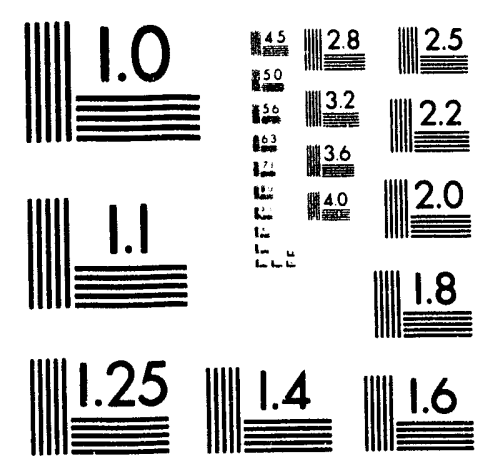



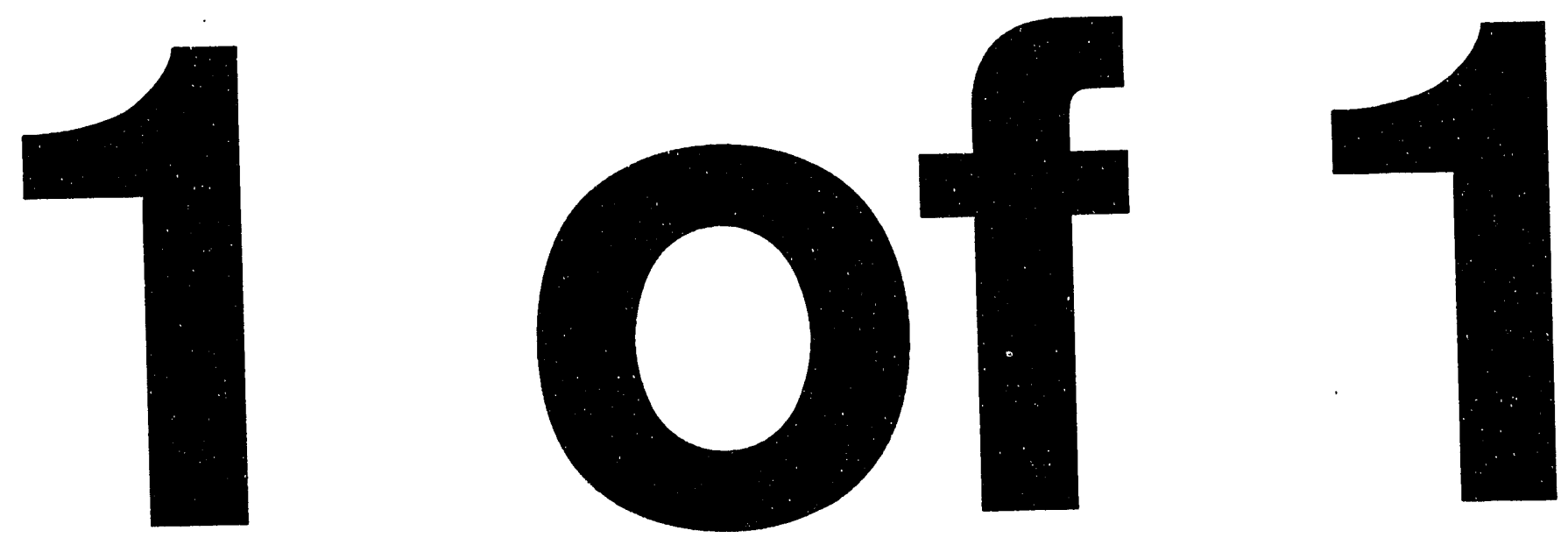


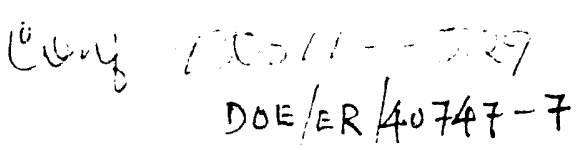

\title{
Even Order Snake Resonances
}

\author{
S.Y. Lee \\ Department of Physics, Indiana University, Bloomington, IN 47405
}

\section{ABSTRACT}

We found that the perturbed spin tune due to the imperfection resonance plays an important role in beam depolarization at snake resonances. We also found that even order snake resonarces exist in the overlapping intrinsic and imperfection resonances. Due to the perturbed spin tune shift of imperfection resonances, each snake resonance splits into two.

\section{Introduction}

The spin equation of motion for a spin particle, governed by the magnetic interaction between the magnetic dipole moment of the particle and the static magnetic field in a synchrotron, is given by the Thomas-BMT equation [1] , $\frac{d \vec{S}}{d t}=\frac{e}{\gamma m} \vec{S} \times\left[(1+G \gamma) \vec{B}_{\perp}+(1+G) \vec{B}_{\|}\right]$, where $\vec{B}_{\perp}$ and $\vec{B}_{\|}$are the transverse and longitudinal components of the magnetic fields with respect to the velocity vector, $\vec{\beta}$. In a planar synchrotron, vertical magnetic fields are needed to guide the orbiting particle around a closed path. Thus the spin vector is precessing with respect to the vertical axis at a frequency $G \gamma f_{0}$, where $f_{0}$ is the revolution frequency, $G=\frac{q}{2}-1$ is the anomalous magnetic $g$-factor and $\gamma$ is the relativistic Lorentz factor. The quantity, $G \gamma$, representing the number of spin precessions per revolution, is called the spin tune.

In a synchrotron, strong quadrupole fields are also needed to focus the beam to a small size. Those particles moving off-center vertically in quadrupoles experience horizontal fields, which will kick the spin vector away from the vertical axis. Since quadrupole magnets and the particle closed orbits are periodic in a circular accelerator and the betatron and the synchrotron motions are quasiperiodic, perturbing kicks to the spin vector can be decomposed into harmonics, $K$, given by $K=n+m \nu_{z}+\ell \nu_{x}+k \nu_{\text {syn }}$, where $\nu_{z}, \nu_{x}$ and $\nu_{s y n}$ are respectively the vertical betatron, the horizontal betatron and the synchrotron tunes, and $k, \ell, m, n$ are integers. The imperfection resonances, due to the vertical closed orbit errors, are located at integer harmonics, $K=n$. The intrinsic resonances, due to the vertical betatron motion, are located at $K=n P+\nu_{2}$, where $P$ is the superperiodicity of the accelerator. Other depolarizing resonance arise from linear or nonlinear betatron coupling vertical dispersion, synchro-betatron coupling and random field errors. When the spin precession frequency is in phase with the harmonics of perturbing kicks, i.e. $G \gamma=K$, these spin perturbing kicks add up coherently every turn around the ring. Therefore the beam can be depolarized.

To avoid a spin resonance condition. Derbenev and Kon- dratenko [3] proposed to use a local spin rotator, which rotates the spin vector $180^{\circ}$ about an axis in the horizontal plane. These spin rotators are called snakes. Using snakes in an accelerator, the spin tune of the particle can become $\frac{1}{2}$ and independent of energy. The resonance condition can be avoided.

\section{Snakes and Spin Motion}

Snakes are local spin rotators, which rotate particle spin by $\pi$ radians about a horizontal axis locally without perturbing particle orbits outside a snake region. A partial snake differs only in the amount of spin rotation angle, e.g. a $10 \%$ snake rotates spin by $0.1 \pi$ radians. Thus a snake is characterized by the amount of spin rotation angle, $\phi$, and the snake axis angle, $\phi_{s}$, with respect to $\hat{e}_{1}$ (radially outward direction). The spinor wave function at a snake will be transformed locally according to $\Psi\left(\theta^{+}\right)=e^{-i \frac{\phi}{2} \hat{n}_{\bullet} \cdot \partial} \Psi\left(\theta^{-}\right)$, where $\phi$ is spin rotation angle and $\hat{n}_{3}=\left(\cos \phi_{s}, \sin \phi_{s}, 0\right)$ denotes the snake axis with respect to radially outward direction, $\hat{e}_{1} \cdot \theta^{ \pm}$depict azimuthal orbit rotation angles just before and after the snake. More specifically, at $\phi=\pi$, or the $100 \%$ snake, the spinor wave function can be transformed as,

$$
\Psi\left(\theta^{+}\right)=e^{-i \frac{\pi}{2} \hat{n}_{1} \cdot \sigma} \Psi\left(\theta^{-}\right)=T_{s}\left(\phi_{0}\right) \Psi\left(\theta^{-}\right),
$$

where $T_{s}\left(\phi_{s}\right)=-i \hat{n}_{3} \cdot \vec{\sigma}$ is the spin transfer matrix for a $100 \%$ snake.

Let us consider a perfect circular accelerator with two snakes, $-i \sigma_{1},-i \sigma_{2}$, separated by $\pi$ orbital angle apart. The one turn spin transfer matrix (OTM) is given by

$$
\left[-i \sigma_{2}\right] e^{-i \frac{a_{\gamma \pi}}{2} \sigma_{3}}\left[-i \sigma_{1}\right] e^{-i \frac{\sigma_{\gamma \pi}}{2} \sigma_{3}}=i \sigma_{3}
$$

Thus the spin tune, obtained from the trace of the one turn spin transfer matrix, is $\frac{1}{2}$ and the stable spin closed orbit is vertical. Now we introduce a small constant local spin angular precessing kick, $\chi$, about an axis $\hat{n}_{k}$ in the horizontal plane, the spin transfer matrix becomes,

$$
T_{1}=e^{-i \frac{x}{2} \hat{n}_{k} \cdot \vec{\sigma}} i \sigma_{3}
$$

Because $\hat{n}_{k}$ is in the horizontal plane, the evolution of the spin transfer matrix at the $n$th revolution becomes,

$$
T^{(n)}=\left[T_{1}\right]^{n}=\left\{\begin{array}{ll}
{\left[i \sigma_{3}\right]^{n}} & \text { if } n=\text { even } \\
T_{1}\left[i \sigma_{3}\right]^{(n-1)} & \text { if } n=\text { odd }
\end{array},\right.
$$

which means that the perturbed spin precessing kicks cancel each other every two turns around the accelerator Thus the snake is effective in correcting imperfection resonances due to a localized constant spin perturbing kick. 


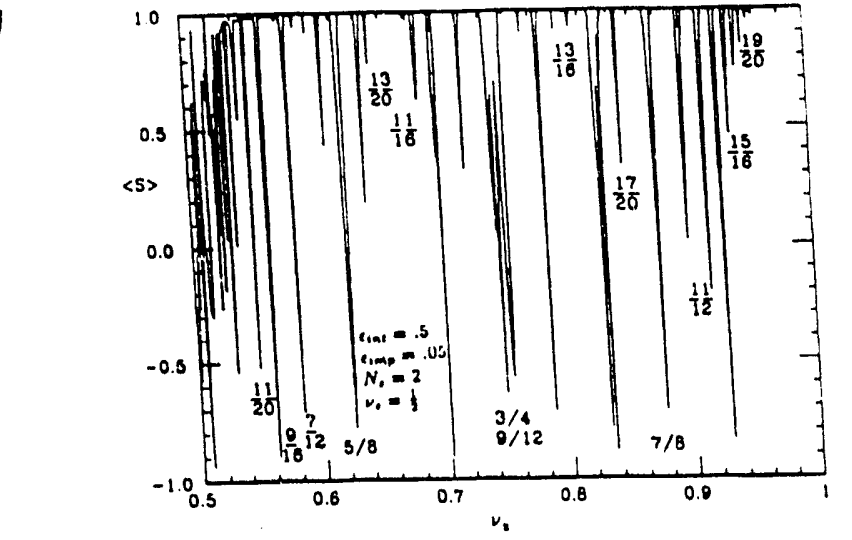

Figure 2: Vertical polarization after passing through an overlapping intrinsic and imperfection resonances with two snakes

two times the betatron frequency with an amplitude proportional to $b^{2} \sin \frac{x}{2}$. Thus the snake resonance condition is given by $\nu_{s} \pm 2 K^{\circ}=$ integer. Performing similar higher order analysis, one obtain all even order snake resonances.

\section{Critical Resonance Strength}

Let us define the critical resonance strength as the resonance strength that the polarization is preserved to within $1.5 \%$ of full polarization. Fig. 3 shows the critical resonance strength for the odd and even order snake resonances at the acceleration rate of $5 \mathrm{MeV} / \mathrm{c}$ per revolution. Depending on the acceleration rate, the critical resonacne strength can be described by the following formula fitted to numerical simulation results,

$$
\begin{aligned}
\epsilon_{c, 5 / 6} & =\ln \left[1.12\left(\frac{\dot{p}}{\dot{p}_{0}}\right)^{0.024}\right] \\
\epsilon_{c, 21 / 26} & =\ln \left[1.64\left(\frac{\dot{p}}{\dot{p}_{0}}\right)^{0.024}\right] \\
\epsilon_{c, 13 / 16} & =\ln \left[1.50\left(\frac{\dot{p}}{\dot{p}_{0}}\right)^{0.020}\right]
\end{aligned}
$$

where the reference acceleration rate is $\dot{p}_{0}=5 \mathrm{MeV} / \mathrm{c}$ per revolution. Here we study only $K=\frac{5}{6}, \frac{13}{16}, \frac{21}{26}$ snake resonances with the assumption that the betatron tune are chosen to lie in between $\frac{4}{5}$ and $\frac{5}{6}$ for example for RHIC at BNL.

\section{Conclusions}

We found that snake resonances, located at $\nu_{s}+\ell K=$ integer, are the major source of depolarization in synchrotrons with snakes, where the integer $\ell$ is called the order of snake resonance, $K$ is the spin resonance harmonics. When imperfection resonances are overlapping with intrinsic resonances, even order snake resonances appear. The perturbed spin tune, arising from imperfection resonances,

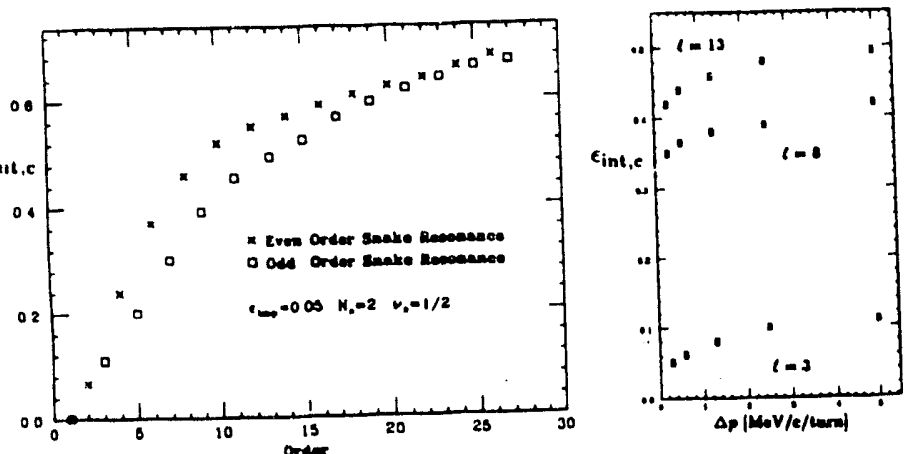

Figure 3: The critical snake resonance strength vs the order of snake resonance is plotted for $\dot{p}_{0}=5 \mathrm{MeV} / \mathrm{c}$ per turn on the left. The critical resonance strength vs the acceleration rate is plotted in the middle part and the snake resonance strength for the even order resonance as a function of the imperfection resonance is shown on the right

plays an essential role in the depolarization mechanism, it causes each snake resonance. to split into two resonances.

\section{References}

1. L.H. Thomas, Phil. Mag. 3, 1 (1927); V.Bargmann, L. Michel, and V.L. Telegdi, Phys. Rev. Lett. 2, 435 (1959).

2. E.D. Courant and H.S. Snyder, Ann. Phys. 3,1(1958).

3. Ya.S. Derbenev, and A.M. Kondratenko, Sov. Phys. Doklady, 20, 562 (1976); Ya.S. Derbenev et al., Particle Accelerators, 8, 115 (1978).

4. S.Y. Lee and S. Tepikian, Phys. Rev. Lett. 56, 1635 (1986); S.Tepikian, Ph. D. Thesis, S.U.N.Y. Stony Brook, (1986), unpublished.

5. A.D. Krisch et al., Phys. Rev. Lett. 63, 1137 (1989). J.E. Goodwin et al., Phys. Rev. Lett. 64, 2779 (1990). J.E. Goodwin, Ph.D. Thesis, Indiana University, (1990), unpublished. M. G. Minty, Ph.D. Thesis, Indiana University, (1991), unpublished. M.G. Minty et al., Phys. Rev. D44, R1361 (1991). M.G. Minty and S.Y. Lee, Proc. EPAC p.723 (1992), and Particle Accelerators, to be published.

6. S.Y. Lee, in Proc. of Workshop on Polarized Beams at SSC, A.D. Krisch ed., AIP Conf. Proc. No.145, p. 189 (1985); E.D. Courant, S.Y. Lee and S. Tepikian, ibid. p. 174 (1985); S.Y. Lee, in High Energy Spin Physics, K.J. Heller ed., AIP Conf. Proc. No.187, p. 1105 (1988).

7. S.Y. Lee and E.D. Courant, Phys. Rev. D41, 292 (1990); S.Y.Lee, Phys. Rev. E, in press.

8. K. Steffen, Particle Accelerator 24, 45 (1989). 


\section{DISCLAIMER}

This report was prepared as an account of work sponsored by an agency of the United States Government. Neither the United States Government nor any agency thereof, nor any of their employees, makras any warranty, express or implied, or assumes any legal liability or responsibility for the accuracy, completeness, or usefulness of any information, apparatus, product, or process disclosed, or represents that its use would not infringe privately owned rights. Reference herein to any specific commercial product, process, or service by trade name, trademark, manufacturer, or otherwise does not necessarily constitute or imply its endorsement, recommendation, or favoring by the United States Government or any agency thereof. The views and opinions of authors expressed herein do not necessarily state or reflect those of the United States Government or any agency thereof. 


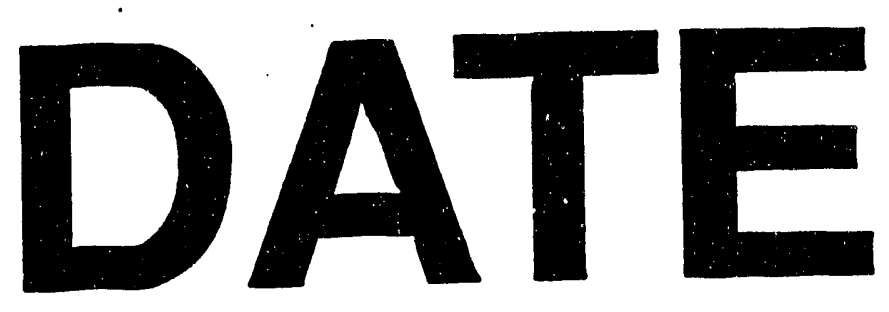

20

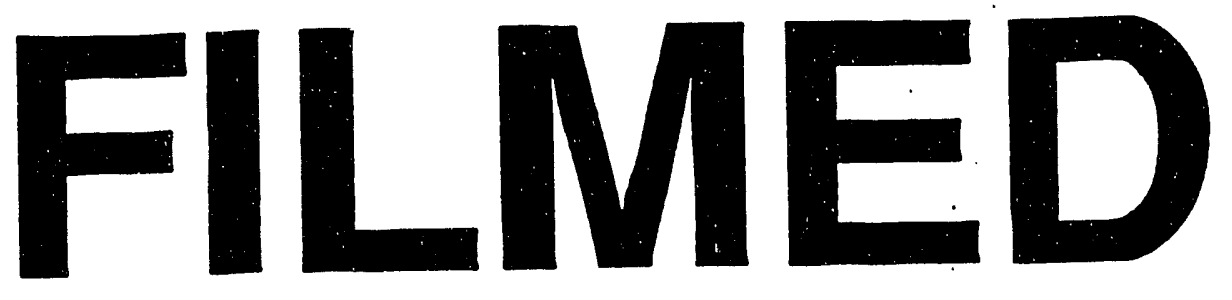

$10 / / 4 / 93$
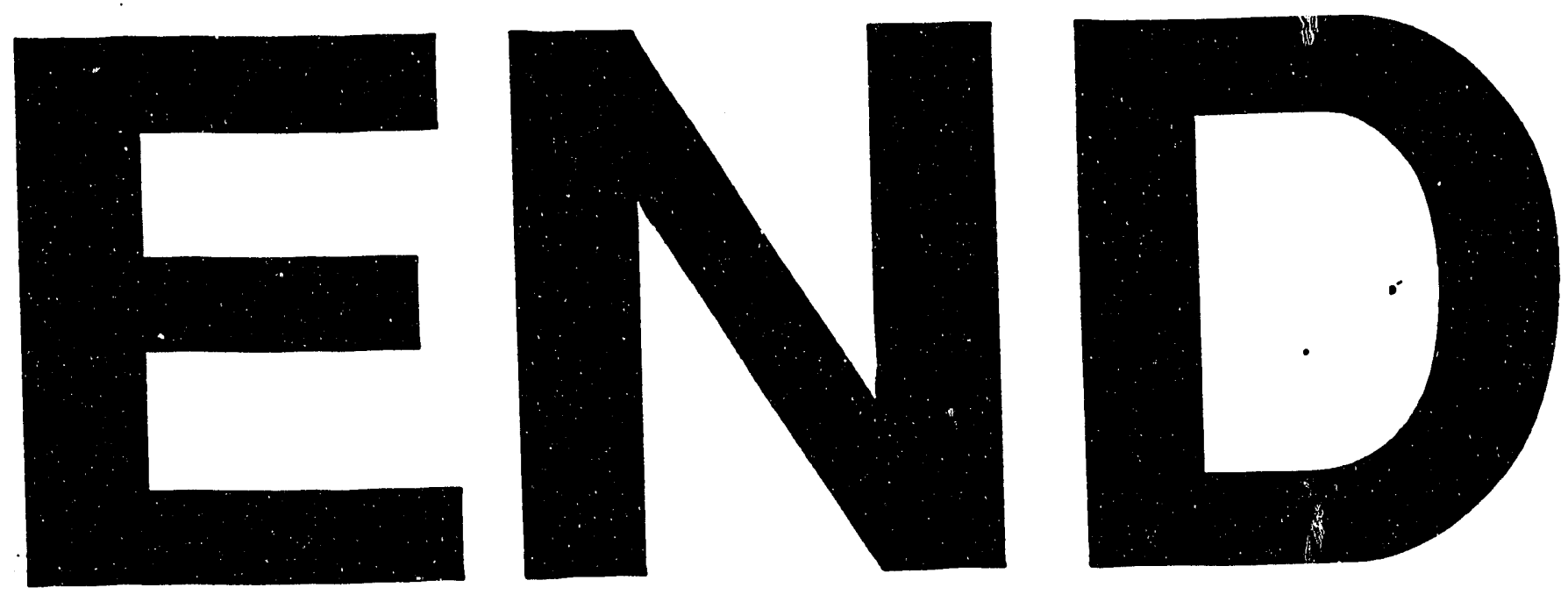
